\title{
Kinetic study of pentosan solubility during heating and reacting processes of steam treatment of green bamboo
}

\author{
Xiaolin Luo a,b,c, Xiaojuan Ma ${ }^{a}$, Huichao $\mathrm{Hu}^{\mathrm{c}}$, Canghai $\mathrm{Li}^{\mathrm{a}}$, Shilin Cao ${ }^{\mathrm{a}}$, Liulian Huang ${ }^{\mathrm{a}}$, Lihui chen ${ }^{\mathrm{a}, *}$ \\ ${ }^{a}$ College of Material Engineering, Fujian Agriculture and Forestry University (FAFU), Fuzhou 350002, China \\ ${ }^{\mathrm{b}}$ Key Laboratory of Biofuel, Chinese Academy of Sciences, Qingdao Institute of Bioenergy and Bioprocess Technology, Qingdao 266101, China \\ ' State Key Laboratory of Pulp and Paper Engineering, South China University of Technology, Guangzhou 510641, China
}

\section{H I G H L I G H T S}

- A modified parameter called steam treatment factor $(f(P))$ was proposed.

- Pentosan in substrates during heating and reacting processes was well predicted.

- Steam treatment factor is a useful tool to compare pretreatment performance.

\section{A R T I C L E I N F O}

\section{Article history:}

Received 19 July 2012

Received in revised form 11 December 2012

Accepted 12 December 2012

Available online 20 December 2012

\section{Keywords:}

Pentosan solubility

Steam treatment

Kinetic model

Steam treatment factor

Green bamboo

\begin{abstract}
A B S T R A C T
Green bamboo was hydrolyzed over a range of durations at different temperatures. A simple pseudohomogeneous irreversible first order kinetic model was developed to describe pentosan solubility during steam treatment of green bamboo. To avoid the influence of soluble pentosan during heating process, kinetic parameters were effectively dissolved based on the data in the reacting process. Moreover, the pentosan solubility during heating process was also well modeled by numerical algorithm method. According to the origin of $\mathrm{H}$ factor, a modified parameter called steam treatment factor $(f(P))$ was proposed in this paper based on the determined kinetic constants. Finally, residual pentosan in whole process could be predicted properly based on the $f(P)$ and the introducing of potential hydrolysis degree $\left(h_{\mathrm{d}}\right)$. After using $f(P)$ to combine reaction temperature and time into a single factor, comparative result showed that steam treatment is more effective for removing pentosan compared with hot water extraction.
\end{abstract}

() 2012 Elsevier Ltd. All rights reserved.

\section{Introduction}

Awareness of climate change and of diminishing fossil fuel reserves has spurred extensive research efforts for bio-fuel production (such as bio-ethanol) from renewable biomass (Himmel et al., 2007). Compared with other renewable lignocelluloses, non-woody materials have attracted increasing attention due to their advantages in terms of abundance and low cost, particularly in countries with a scarcity of wood (Sundqvist, 2001). As one of the most important non-woody resources, bamboo is a perennial species and is distributed widely in Asia (Scurlock et al., 2000). Due to its fast growth (3-5 years), easy propagation, high productivity, and high holocellulose content (64-70\%), bamboo is one of the ideal raw materials for the production of bio-fuels, such as ethanol (Shimokawa et al., 2009) and methanol (Tsuda et al., 1998). However, due to the tree-like structure of bamboos, the celluloses

\footnotetext{
* Corresponding author. Tel.: +86 591 83715175; fax: +86 59183789495 .

E-mail address: luoxiaolin128@yahoo.com.cn (L. chen).
}

are tightly connected with other main components, particularly hemicelluloses. For this reason, enzymatically hydrolyzing bamboo cellulose into fermentable sugar is very difficult (Sathitsuksanoh et al., 2010). Therefore, the pretreatment for removing the hemicelluloses in bamboo is necessary for the production of sugars via enzymatic saccharification.

Among many practical technologies, steam explosion (Mason, 1929 ) is regarded as one of the most effective pretreatment methods for non-woody resources, such as corn stover (Alvira et al., 2010; Saddler et al., 1993). Although the effectiveness of such pretreatment, including those integrated with chemical reaction and mechanical treatment, on the substantial enzymatic hydrolysis has been fully investigated (Dekker et al., 1987; Kling et al., 1987; Ramos et al., 1992; Tanaka and matsuno, 1990), the kinetic study of hemicelluloses solubility during the steam treatment process alone has been few reported.

Understanding the kinetics of hemicellulose hydrolysis is extremely important for testing mechanisms, process control, and optimization during hydrothermal and steam pretreatment 
of lignocellulosic feedstock (Abatzoglou et al., 1992; Brennan and Wyman, 2004; Lu and Mosier, 2008; Springer, 1966; Zhao et al., 2012; Zhu et al., 2012a). The published models that delve into the solubility of hemicelluloses (mainly pentosan) can be divided into two categories: the homogeneous pseudo-first-order model (Aguilar et al., 2002; Saeman, 1945) and the biphasic reaction model that incorporates fast (easy) and slow (difficult) hydrolyzing fractions (Kobayashi and Sakai, 1956; Maloney et al., 1985; Zhao et al., 2012; Zhu et al., 2012a). Some deviations from both classic models have been proposed, and these models take into consideration the intermediates (Abatzoglou et al., 1992), the mass transfer effect (Brennan and Wyman, 2004; Mittal et al., 2009) or catalysts (Zhu et al., 2012a). For example, Zhu et al. (2012a) proposed a combined hydrolysis factor (CHF) considering the effect of catalysts by dilute acid and two sulfite pretreatments at different $\mathrm{pH}$. However, the model was simply developed based on the hypothesis of that the whole process could be simply regarded as an isothermal process. Detailed kinetic analysis of heating process was lacked. Likewise, if there is no uniform severity factor which can conclude the heating and reacting processes during chemical pretreatment, the different efficiency in removing pentosan between non-isothermal steam and hydrothermal treatments cannot be thoroughly compared. Moreover, data on the concentrations of xylo-oligomers, xylose, and inhibitors used to determine the kinetic constants in the aforementioned models mostly originate from measurements in the liquid phase (Jensen et al., 2008; Lu and Mosier, 2008). But measuring all degraded products is not feasible (Zhao et al., 2012). Although Zhao et al. (2012) obtained kinetic parameters based on the data measured in the treated solids after dilute acid pretreatment, the temperature range was limit (only from 97 to $126^{\circ} \mathrm{C}$ ). Unfortunately, low severity pretreatments, especially steam treatment, are normally less satisfactory to subsequent enzymatic hydrolysis for woody or some non-woody biomass. Furthermore, the ratio of easy-to-hydrolyze and difficult-to-hydrolyze fractions of pentosan (also regarded as potential hydrolysis degree, $h_{\mathrm{d}}$ ) is also not determined during steam treatment.

Thus, the present study attempts to address the shortcomings discussed above. The objectives of this study are (1) to address the effect of heating-up period on kinetic analysis of pentosan solubility during steam treatment of green bamboo; (2) to effectively fit pentosan solubility during heating-up process; and (3) to compare the pentosan solubility efficiency between steam and hydrothermal treatments based on the modified severity factor.

\section{Methods}

\subsection{Raw materials}

The green bamboo (Dendrocalamopsis oldhami (Munro) Ke) chips used in the experiments were generously provided by Nanjing Forestry (Zhangzhou City, Fujian, China). The chips were air-dried and screened to a relatively homogeneous size of $30 \mathrm{~mm} \times 15 \mathrm{~mm} \times$ $3 \mathrm{~mm}$. The screened chips were ground to pass a 40-mesh screen using a grinder for chemical composition analysis. The composition of the green bamboo was determined as follows: $49.6 \% \alpha$-cellulose, $17.5 \%$ pentosan, $23.1 \%$ klason lignin, $6.8 \%$ hot-water extracts, and $1.8 \%$ ash on a dry weight basis.

\subsection{Steam treatment}

Steam treatment was conducted in a batch steam explosion apparatus equipped with a $1.2 \mathrm{~L}$ reactor $(\mathrm{NK}-1 \mathrm{~L}$, Japan Chemical Engineering and Machinery Co. Ltd., Osaka, Japan). Approximately $100 \mathrm{~g}$ o.d. of screened green bamboo chips was introduced to the reactor. The reactor was then heated internally with steam to the desired temperature $\left(160^{\circ} \mathrm{C} / 0.6 \mathrm{MPa}, 170^{\circ} \mathrm{C} / 0.8 \mathrm{MPa}, 180^{\circ} \mathrm{C} /\right.$ 1.0 MPa, and $190^{\circ} \mathrm{C} / 1.2 \mathrm{MPa}$ ) and maintained there for $0-70 \mathrm{~min}$. The temperatures $(T)$ were directly recorded by thermocouple, which was located in $2 / 3$ height of the internal of reactor. The inside of the reactor is looked as oval with the diameter and height of approximately 5 and $15 \mathrm{~cm}$, respectively. All temperatures $(T)$ used in this study present the temperatures shown on the thermocouple. After the steam process, the pressure was slowly released into a container to avoid the effect of explosion on the subsequent process. The solids and liquids were separated in the Buchner funnel with a filter paper (diameter of $12.5 \mathrm{~cm}$, slow type, Xinhua Corporation, Hangzhou, China). Bamboo chips that were treated with steam without explosion were washed three times using tap water to remove soluble sugars before being subjected to the hydrolysis experiments. The solids were collected and then stored in sealed plastic bags at $4{ }^{\circ} \mathrm{C}$ for additional experiments.

\subsection{Analytical methods}

The solid yield was determined by the weight and moisture content of the steam-treated bamboo chips. The moisture content was determined gravimetrically by drying a portion of the collected solids in an oven at $105^{\circ} \mathrm{C}$ overnight. The pentosan contents of bamboo chips after steam treatment were determined in accordance with the TAPPI Standard (T $223 \mathrm{~cm}-01,2001$ ). Pentosans are transformed in boiling $3.85 \mathrm{~mol} / \mathrm{l}$ hydrochloric acid to furfural, which are collected in the distillate and determined colorimetrically with orcinol-ferric chloride reagent.

The fraction of residual pentosan in solid substrate $(\mathrm{P})$ was determined from the measured pentosan content, $C_{P m}$, and the solid substrate yield, $Y s$.

$P=\left(C_{P m} \times Y_{S}\right) / C_{P 0}$.

\subsection{Data analysis}

The kinetic constants and other parameters were fitted according to the experimental data by minimizing the sum of squares using commercial software with a built-in optimization routine that is based on Newton's method (Solver, Microsoft Excel). The experimental and calculated data are shown in Figs. 2 and 3.

\section{Results and discussion}

\subsection{Effect of steam treatment temperature and time on pentosan solubility}

Pentosan solubility during steam treatment was studied in a wide range of reaction conditions. Thirty-two experiments were carried out at $160-190{ }^{\circ} \mathrm{C}$ for different durations. The residual fraction of pentosan in the bamboo chips (based on o.d. of untreated bamboo chips) at each reaction temperature is shown in Fig.1. The fraction of soluble pentosan increased with the increasing duration of the steaming treatment. The residual fraction of pentosan in bamboo chips decreased from about 0.111 to 0.067 when the duration was extended from $10 \mathrm{~min}$ to $70 \mathrm{~min}$. The results concurred with those obtained by Ma et al. (2011) under identical conditions of hot-water extraction with the same bamboo chips. Ma et al. (2011) reported a maximum pentosan extraction yield of close to $26.8 \%$ in a hot-water extraction of the same bamboo chips at $170^{\circ} \mathrm{C}$ after 60 min reaction. Moreover, their results showed that the soluble fraction of pentosan reached $64.9 \%$ at $170{ }^{\circ} \mathrm{C}$ after 120 min of extraction. However, in the present study, at $170{ }^{\circ} \mathrm{C}$, about $58 \%$ of the initial pentosan in the bamboo sample was extracted after 60 min of steaming (Fig.1), indicating the effectiveness of direct steam treatment in accelerating the 


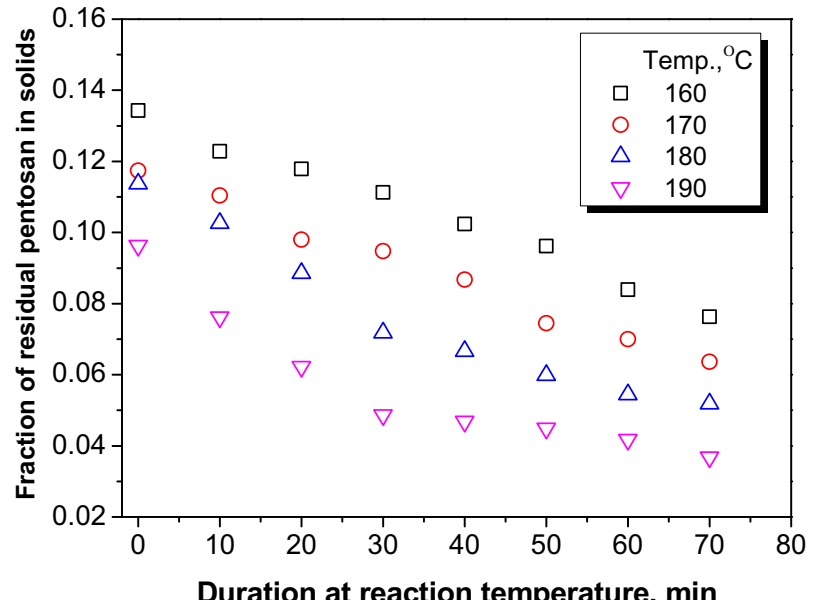

Fig. 1. Change in residual pentosan of bamboo solids during steam treatment at four reaction temperatures. (Note: the fraction of residual pentosan in steamtreated bamboo solids were calculated based on o.d. weight of untreated bamboo chips.)
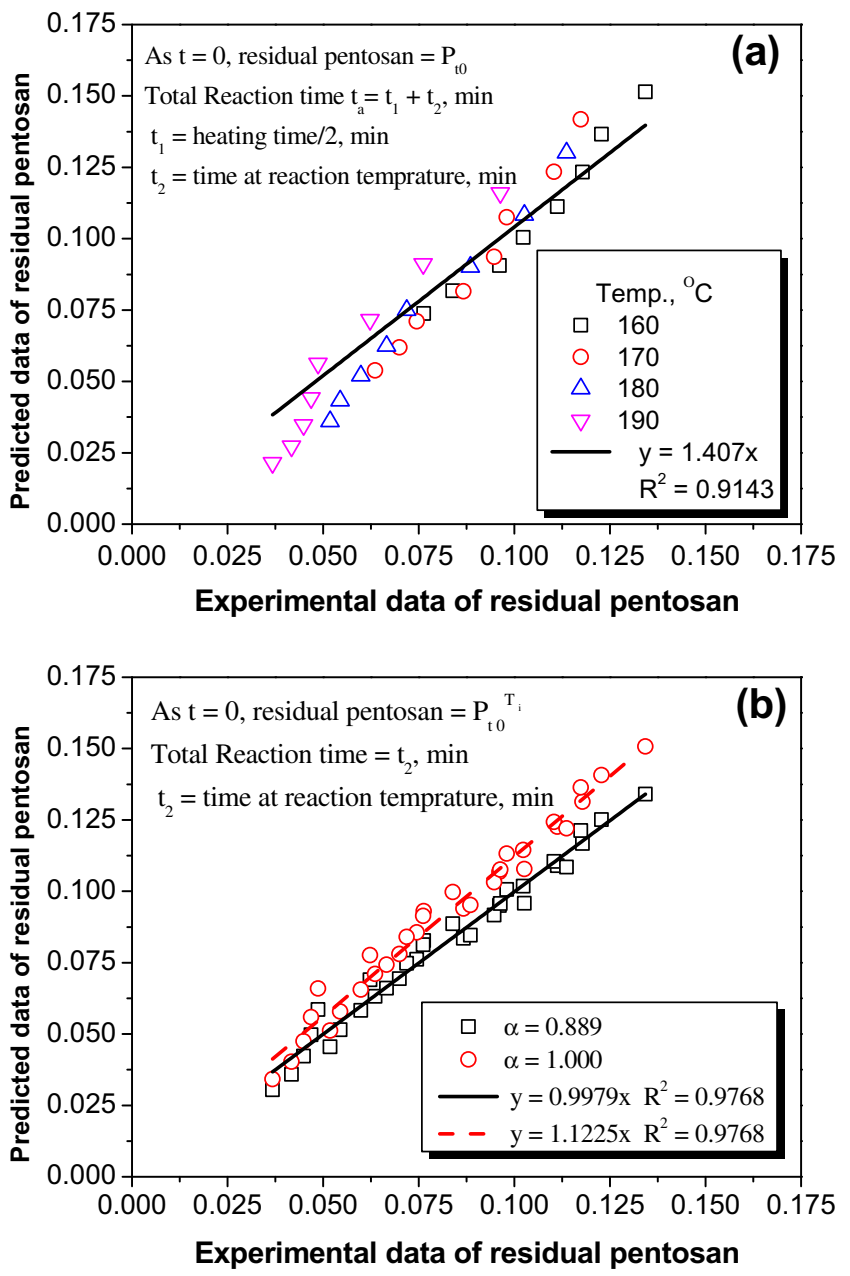

Fig. 2. Comparison of predicted data with experimental data for residual pentosan of green bamboo during steam treatment. (a): Traditional model (Eq. (6)); (b): Modified models (Eqs. (8) and (9)).

process. Steam treatment could preferentially soften the wall structure of lignocellulosic materials and then increase the solubility of pentosan.
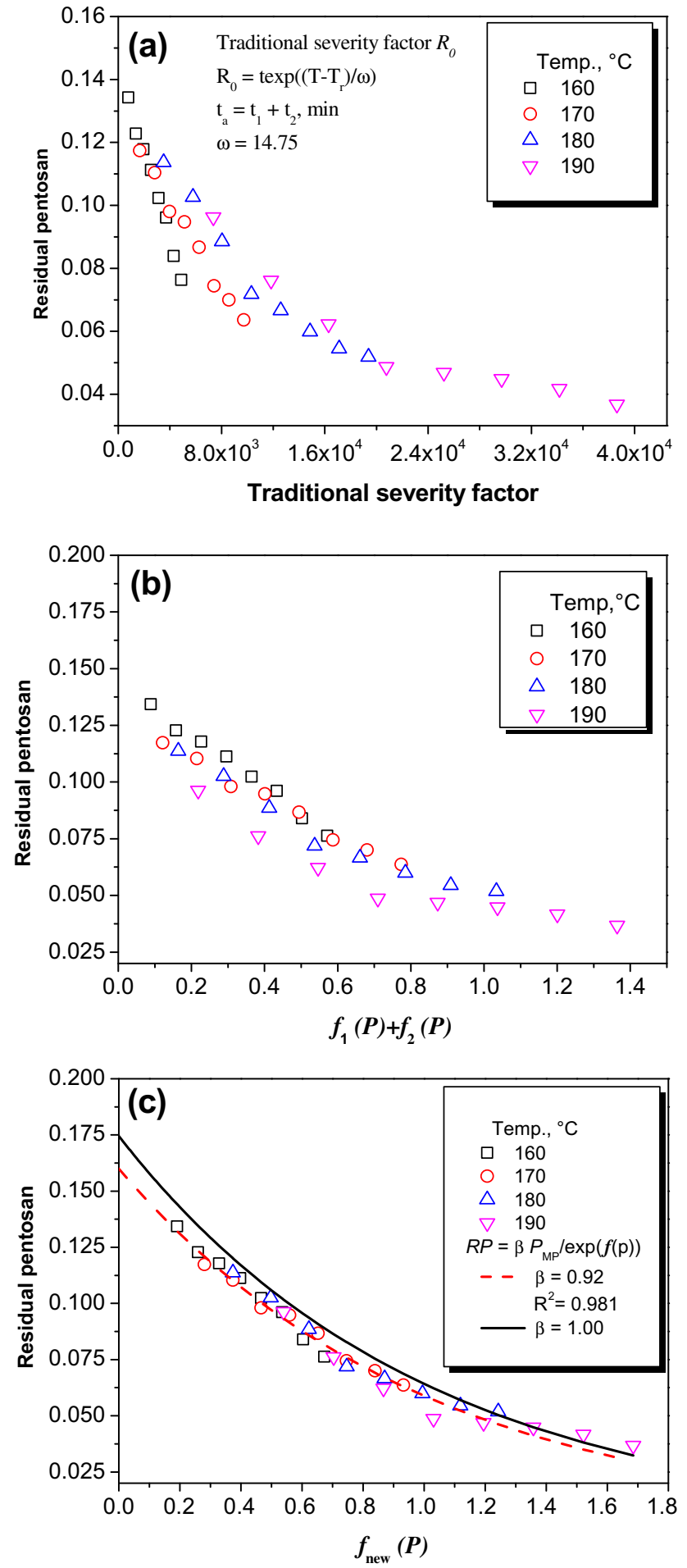

Fig. 3. Correlation between residual pentosan from different steam treatment conditions and the steam treatment factor $(f(P))$. (a) Calculating $f(P)$ in the heating process using the numerical algorithm; (b) Taking the saturated steam temperature as reaction temperature during the heating process and introducing the potential hydrolysis degree $\left(h_{\mathrm{d}}\right)$ into the model (Eq. (20)).

Earlier, the steam treatment was conducted in a non-isothermal operation during heating-up period; hence, part of the pentosan was degraded during the heating process. Then, the reaction goes into a constant $\mathrm{T}$ (isothermal) period. Moreover, the results obtained in the reaction process indicated that the reaction rate increased substantially with the increase in temperature. However, 
at elevated temperatures $\left(180-190^{\circ} \mathrm{C}\right)$, it was found in Fig. 1 that the rate of pentosan removal become slow after the reaction time beyond $50 \mathrm{~min}$. These data confirm that, although reaction temperature and reaction time both influence the hydrolysis of bamboo pentosan considerably, not all pentosan in the bamboo chips could be removed in the reaction temperature range used in this study.

\subsection{Evaluation of mass transfer effects}

The Weisz modulus $\left(M_{\mathrm{W}}\right)$ represents the ratio of the reaction rate and diffusion rate, and it can be used to evaluate the internal particle mass transfer limitation. It can be expressed as

$M_{\mathrm{W}}=\frac{\mathrm{r}_{p}}{C_{\mathrm{H}^{+}, \mathrm{S}}} \frac{\left(d_{b} / 6\right)^{2}}{D_{\mathrm{H}^{+}, s}}$

where $r_{\mathrm{p}}$ is the reaction rate of pentosan removal, in $\mathrm{mol} /\left(\mathrm{m}^{3} \mathrm{~s}\right)$; $d_{b}$ is the vertical thickness of bamboo chips, in $\mathrm{m}$; and $\mathrm{C}_{\mathrm{H}+\mathrm{S}}$ and $D_{\mathrm{H}}^{+}$, $\mathrm{s}$ are the concentration and the diffusion coefficient of $\mathrm{H}^{+}$in the solid phase, expressed in $\mathrm{mol} / \mathrm{m}^{3}$ and $\mathrm{m}^{2} / \mathrm{s}$, respectively.

Fig. 1 shows that $45 \%$ of pentosan was removed at $170{ }^{\circ} \mathrm{C}$ within $30 \mathrm{~min}$. The density of green bamboo is about $1.00 \mathrm{~g} / \mathrm{cm}^{3}$ (Chu, 2005). Thus, the $r_{\mathrm{p}}$ was calculated to be $1.645 \times 10^{-6} \mathrm{~mol} /\left(\mathrm{m}^{3} \mathrm{~s}\right)$.

According to Malm et al. (1952), the concentration of $\mathrm{H}^{+}$in solid substrate is eight times that in the liquid phase. The $\mathrm{pH}$ of the hydrolyzate, sampled at $170{ }^{\circ} \mathrm{C}$ for $30 \mathrm{~min}$, was measured to be 4.25 , and the value of $\mathrm{C}_{\mathrm{H}+\mathrm{s}}$ was calculated to be $0.450 \mathrm{~mol} / \mathrm{m}^{3}$.

In a porous medium, the following expression can be used to estimate the diffusion coefficient of $\mathrm{H}^{+}$in the solid substrate:

$D_{\mathrm{H}^{+}}$, solid $=D_{\mathrm{H}^{+}}$, water $\psi^{1.5}$

where $\psi$ and $D_{\mathrm{H}^{+}}$, water denote the void fraction (\%) of bamboo chips and the diffusion coefficient $\left(\mathrm{m}^{2} / \mathrm{s}\right)$ of $\mathrm{H}^{+}$in water, respectively.

The void fraction of bamboo species normally ranges from $20 \%$ to $40 \%$ (Chu, 2005). Using an average void fraction of $30 \%$ and a diffusion coefficient of $1.8 \times 10^{-9} \mathrm{~m}^{2} / \mathrm{s}$ for $\mathrm{H}^{+}$in water, the value of $\mathrm{D}_{\mathrm{H}+}$, solid was estimated to be $2.96 \times 10^{-10} \mathrm{~m}^{2} / \mathrm{s}$.

The mass transfer limitation is reportedly insignificant if the Weisz modulus $\left(M_{\mathrm{W}}\right)$ does not exceed 0.15 (Levenspiel, 1999). With the aforementioned data and an average bamboo chip vertical thickness of $3 \mathrm{~mm}$, the $M_{\mathrm{W}}$ was estimated as approximately $1.03 \times 10^{-3}$, which is far less than 0.15 . This finding indicates that the diffusion limitation for the steam treatment of green bamboo at $170{ }^{\circ} \mathrm{C}$ for $30 \mathrm{~min}$ is absent. According to the liquid-to-solid ratio obtained in Section Supplemental A, the concentration and diffusion coefficient of $\mathrm{H}^{+}$in the solid phase at four reaction temperatures will increase or decrease by $20 \%$. Hence, the value of $M_{\mathrm{W}}$ for all experiments will stay in the same order of magnitude at $<<0.15$. Therefore, the mass transfer limitation would not affect the kinetic data of pentosan removal during the steam treatment of green bamboo.

\subsection{Development and solution of kinetic models}

The mechanism for hemicellulose (especially pentosan) hydrolysis is not accurately modeled because of its complex structure and inability to analyze degradation products completely. In this study, pentosan hydrolysis during steam treatment was also based on the hypothesis of pseudo-homogeneous kinetic reactions (Abatzoglou et al., 1992; Kobayashi and Sakai, 1956; Saeman, 1945). This simplified scheme does not take into account the formation of monosaccharides and other possible by-products as they are not the focus of this study. Therefore, the overall scheme of pentosan solubility during steam treatment can be generalized as
Pentosan(in solid) $+\mathrm{H}_{2} \mathrm{O} \rightarrow$ Pentosan $\quad$ (in liquid) $+\mathrm{H}^{+}$

The simple pseudo-homogeneous irreversible first order kinetic equation (Jensen et al., 2008; Lu and Mosier, 2008) used to describe the rate of hemicellulose hydrolysis during the acidic or hydrothermal pretreatment of lignocelluloses is defined as

$-\frac{d P}{d t}=k_{0} e^{-\frac{E a}{R T} P}$

where $P$ is the remaining pentosan content (based on o.d. of the bamboo chips) in solid at time $t, E_{a}$ represents the activation en$\operatorname{ergy}(\mathrm{J} / \mathrm{mol}), k_{0}$ is the frequency factor $\left(\mathrm{min}^{-1}\right), R$ is the universal gas constant $(\mathrm{J} / \mathrm{mol} \mathrm{K})$, and $T$ is the absolute temperature $(\mathrm{K})$.

Some of the pentosan dissolved during the initial process of heating, which is a non-isothermal operation. Based on Zhu et al. (2012a), the current work assumed that half the ramping time can be equivalent to the reaction time. Firstly, the heating-up and constant $\mathrm{T}$ periods were taken as a whole process to do the kinetic analysis. Therefore, the total reaction time $t_{\mathrm{a}}=t_{\text {reaction }}+$ $t_{\text {heating }} / 2$.

Integrating Eq. (4) yields

$\int_{P_{\mathrm{t} 0}}^{P_{t}} \frac{d P}{P}=-k_{0} e^{-\frac{E a}{R T}} \int_{0}^{t} d t_{\mathrm{a}}$

The solution to Eq. (5) for Pt gives

$P_{t}=P_{\mathrm{t}_{0}} \exp \left(-k_{0} e^{-\frac{E a}{R T}} t_{\mathrm{a}}\right)$

where $P_{\mathrm{t}_{0}}$ is the pentosan content in bamboo chips.

The estimates of the kinetic parameters in the traditional model (Eq. (6)) were determined using the least squares method. The results are given in Table 1 . The predicted data on remaining pentosan in solid did not exactly correlate to the experimental with $s^{2}=0.81$. Fig. 2(a) shows a comparative plot that confirms the mismatch. Therefore, the simple equivalent approach of ramping time is not applicable when describing the kinetic pentosan solubility of green bamboo during steam treatment.

As short heating time used in Zhao et al. (2012) and Zhu et al. (2012a), ignoring or simple treatment of ramping time have no big effect on kinetic analysis. However, this method is deficient for green bamboo pentosan hydrolysis during steam treatment. Therefore, the whole pentosan hydrolysis process could be divided into heating-up and constant $\mathrm{T}$ periods. In order to overcome the problem caused by non-isothermal process (pentosan hydrolyzed during heating-up period), the kinetic model can be developed based on the data obtained in the reaction process alone. Hence, the pentosan content in steam treated solids for zero reaction time at each constant $T$ was initially determined. The remaining pentosan linearly decreased with the increase of reaction temperature (Supplemental B). The relationship between pentosan and reaction temperature can be expressed as

$P_{t_{0}}^{T_{i}}=a\left(T_{i}-T_{0}\right)+b$

Thus, in kinetic analysis, the pentosan contents in steam treated solids were deducted the dissolved pentosan during heating-up period. Finally, the kinetic model in the process of constant $T$ was modified as

Table 1

Kinetic constants for pentosan solubility in the traditional and modified models.

\begin{tabular}{lll}
\hline & Traditional model & Modified model \\
\hline $\mathrm{k}_{0}, \mathrm{~min}-1$ & $3.82 \mathrm{E}+04$ & $4.33 \mathrm{E}+03$ \\
$\mathrm{Ea}, \mathrm{kJ} / \mathrm{mol}$ & 53.96 & 48.06 \\
$\mathrm{a}$ & None & -0.0014 \\
$\mathrm{~b}$ & None & 0.3448 \\
$\alpha$ & None & 0.889 \\
$\mathrm{R}^{2}$ & 0.807 & 0.977 \\
\hline
\end{tabular}


$\int_{P_{t_{0}}^{T_{i}}}^{P_{t}} \frac{d P}{P}=-k_{0} e^{-\frac{E a}{R T}} \int_{t_{0}}^{t} d t_{\text {reaction }}$

where $P_{t_{0}}^{T_{i}}$ represents the remaining pentosan in the solid for zero reaction time at each constant $T$; the $i$ in $T_{i}$ can be regarded as $1,2, \ldots ; \mathrm{a}$ and $\mathrm{b}$ are constants, and $T_{\mathrm{i}}$ and $T_{0}$ denote the reaction and room temperatures (K), respectively.

In verifying this modified model, the predicted data were further compared with the experimental data on the steam treatment of green bamboo (Fig. 2(b)). However, although the square of correlation coefficient between the experimental and predicted data was very high (0.977), the slope of fitting of the linear line was more than 1 . The remaining pentosan in the bamboo solid was thus overestimated by Eq. (8). The apparent deviation here may be due to the potential hydrolysis degree $\left(h_{\mathrm{d}}\right)$ reported by Garrote et al. (2002) and Zhao et al. (2012), whereby a small portion of pentosan remains in lignocelluloses during thermal-chemical pretreatment. The $h_{\mathrm{d}}$ is dependent on the severity of the reaction and the catalyst used in pretreatment. Two main reasons have been used to explain the introduction of $h_{\mathrm{d}}$ into the kinetics of pentosan hydrolysis: the wide distribution of pentosan in the cell wall and the complex linkages between hemicelluloses with the lignin and cellulose. Due to the fitting deviation observed in the traditional kinetic model (Eq. (6)) that was similar to that in the studies of Garrote et al. (2002) and Zhao et al. (2012), the $h_{\mathrm{d}}$ was also introduced into the reaction kinetics of pentosan solubility during the steam treatment of green bamboo. Finally, Eqs. (7) and (8) were combined, with consideration for the $h_{\mathrm{d}}$, and the time-dependent expression for $P_{t}$ was generated as follows:

$P_{t}=\alpha\left[a\left(T_{i}-T_{0}\right)+b\right] \exp \left(-k_{0} e^{-\frac{E a}{R T}} t_{\text {reaction }}\right)$

where $\alpha$ is the potential hydrolysis degree of pentosan, $0 \leqslant \alpha \leqslant 1$.

Fig. 2(b) presents a comparison of the pentosan solubility values obtained from the proposed novel kinetic model (Eq. (9)) with the experimental data. The slope was very close to 1 , and the square of correlation coefficient $\left(R^{2}\right)$ was kept at a high level of 0.977 . Thus, the modified model fitted the experimental data appropriately. The fitted constants are shown in Table 1. Compared with the kinetic results in the traditional model, the modified model exhibited a decrease in the activation energy $(48.1 \mathrm{~kJ} / \mathrm{mol})$ and frequency factor $\left(4330 \mathrm{~min}^{-1}\right.$ ) after using $h_{\mathrm{d}}$. However, these constants, which were determined in the modified model, were of the same order as those in most literature on pentosan hydrolysis of non-woody feedstock (Arslan and Saraçoğlu-Eken, 2012; Lavarack et al., 2000; Romero et al., 2010).

\subsection{Correlation of pentosan solubility with modified severity factor}

\subsubsection{Predicting pentosan solubility using the modified severity factor}

Many research (Abatzoglou et al., 1992; Garrote et al., 2002) found that traditional severity factor can combine reaction temperature and time into a single factor and thus fit the pentosan removal well during acidic chemical pretreatment of biomass. To verify the applicability of traditional severity factor in this study, this factor was used to fit the pentosan solubility during steam treatment of green bamboo. The spread of experimental data was found in Fig.3(a). This mainly results from the different activation energy of pentosan hydrolysis, comparing to pentosan removing from woody biomass during dilute acid or hydrothermal pretreatment.

The modified kinetic model in Section 3.3 could provide the activation energy and frequency factor accurately. However, predicting pentosan solubility in the process of heating is difficult due to the development of this kinetic model that is based mainly on data in the reaction process. As with industrial chemical pulping, the heating process would be involved in the commercial thermal-chemical pretreatment or fractionation of lignocelluloses for biofuel production. Therefore, one parameter, such as the $\mathrm{H}$ factor (Vroom, 1957), is expected to combine both reaction temperature and time into a single factor; the effects of heating and reacting processes on biomass pretreatment must also be included. Based on the derivation of the $\mathrm{H}$-factor, a differential equation of pentosan solubility can be expressed as

$-\frac{d P_{t}}{p_{t}}=k_{0} e^{-\frac{E a}{R T}} d t$

where

$-\int_{P_{t_{0}}}^{P_{t}} \frac{\mathrm{d} P_{t}}{p_{t}}=f(P)$

and

$f(P)=\int_{0}^{t} k_{0} e^{-\frac{E a}{R T}} d t$

For the non-isothermal process in steam treatment, temperature can be simply estimated as linear growth in a very short time, according to the numerical algorithm. Taking the time step of $0.1 \mathrm{~min}$, the reaction temperature can be expressed as

when $T_{0}=25, \quad T_{1}=T_{0}+v \Delta t$

thus,

$T_{i}=T_{i-1}+v \Delta t$

where $v$ is the heat rate $\left({ }^{\circ} \mathrm{C} / \mathrm{min}\right)$, and $\Delta t$ is the step ( $\left.\mathrm{min}\right)$.

The combination of Eqs. (11) and (12) with the numerical algorithm of Eq. (11) leads to the following expressions:

$f_{1}\left(P_{1}\right)=k_{0} e^{-\frac{E a}{R\left(T_{0}+T_{1}\right) / 2}} \Delta t$,

$f_{1}\left(P_{i}\right)=\sum_{i=1}^{i} k_{0} e^{-\frac{E a}{R\left(T_{i}+T_{i-1}\right) / 2}} \Delta t$

where $i=t_{\text {heating }} / \Delta t$.

For the isothermal process in steam treatment, $d_{t}=t_{\text {reaction, Eq. }}$ (11) can be derived as

$f_{2}(P)=k_{0} e^{-\frac{E a}{R T}} t_{\text {reaction }}$

A novel parameter, called steam treatment factor, $f(P)$, which combines $f_{1}(P)$ and $f_{2}(P)$, was proposed to describe the profile of pentosan solubility during steam treatment.

$f(P)=f_{1}(P)+f_{2}(P)$

Using the activation energy and frequency factor obtained in Section 3.3, $f(P)$ was used to reflect the effects of temperature and time on pentosan dissolution from the heating process to the reacting process of steam treatment. After plotting $f(P)$ with the residual pentosan from each reaction temperature, a simple exponential correlation between both of them could be obtained:

$R P=\frac{P_{t_{0}}}{e^{f(P)}}$

where $R P$ represents the remaining pentosan in bamboo chips.

However, the experimental data of residual pentosan at different reaction temperatures were not on a single curve (Fig.3(b)). All data from four reaction temperatures would fall on the same curve (Fig.3(c)) if the heating process is equivalent to the insulation process. This assumption resulted in the following expressions:

$t=t_{\text {heating }}+t_{\text {reaction }}$

$f_{\text {new }}(P)=k_{0} e^{-\frac{E a}{R T}}\left(t_{\text {heating }}+t_{\text {reaction }}\right)$ 
The following processes were assumed to occur: (1) at the beginning of steam injection, the saturated steam rapidly penetrated the surface and interior of the bamboo; (2) the water molecules in situ immediately reacted with pentosan, where the reaction temperature was equal to the temperature of saturated steam; (3) the hot saturated steam began to condense into liquid after heat exchange, and partial internal space in the cell wall of the bamboo chip gave out; (4) the fast entry of fresh steam led to the start of a new cycle until the reaction system entered the isothermal process.

Therefore, the reaction temperature for pentosan hydrolysis in the heating process can be regarded as the temperature in constant $T$ (equal to saturated steam temperature). Without the introduction of the potential hydrolysis degree $\left(h_{\mathrm{d}}\right)$ discussed in Section 3.3 to Eq. (17), the initial conditions obtained would be $\lim f(p) \rightarrow+\infty$ $R P=0$ and $\lim _{(} f(p) \rightarrow 0 R P=P_{t 0}$. Again, the residual pentosan data could be fitted with $f_{\text {new }}(P)$ by setting $\beta$ as 1 . The fitting results in Fig. 3(b) show an overestimation of residual pentosan. On the contrary, by applying the potential hydrolysis degree $\left(h_{\mathrm{d}}\right)$ in predicting the residual pentosan using $f_{\text {new }}(P)$, Eq. (17) may be rewritten as

$R P=\frac{\beta P_{t_{0}}}{e^{f_{\text {new }}(P)}}$

After fitting the experimental data of residual pentosan with $f_{\text {new }}(P)$ without setting $\lim f(p) \rightarrow 0 R P=P_{t 0}$, the parameter of $\beta$ was deduced as 0.92 for Eq. (20). Fig. 3(b) shows that the experimental data can be aptly predicted by Eq. (20) with $r^{2}=0.981$, indicating that the potential hydrolysis degree $\left(h_{\mathrm{d}}\right)$ is suitable for the steam treatment of green bamboo under certain conditions. The values of $h_{\mathrm{d}}$ for pentosan removal of green bamboo during steam treatment obtained in Eqs. (9) and (20) were also consistent, considering the solubility of pentosan during the heating process in Section 3.3. Thus, these models are reliable for confirming the existence of the potential hydrolysis degree $\left(h_{\mathrm{d}}\right)$ for the solubility of pentosan in green bamboo under certain steam treatment conditions.

\subsubsection{Relationship between the traditional severity factor and the steam treatment factor}

The severity factor $\left(R_{0}\right)$ in Eq. (21) has often been used to describe the hydrothermal pretreatment of lignocelluloses for biofuel production (Garrote et al., 2002; Zhao et al., 2012; Zhu and Zhuang, $2012 \mathrm{~b}$ ). The relationship between $f_{\text {new }}(P)$ and $R_{0}$ for the experimental conditions conducted in this study was explored. Fig. 4 shows that $f_{\text {new }}(P)$ is linearly correlated to $R_{0}$ :

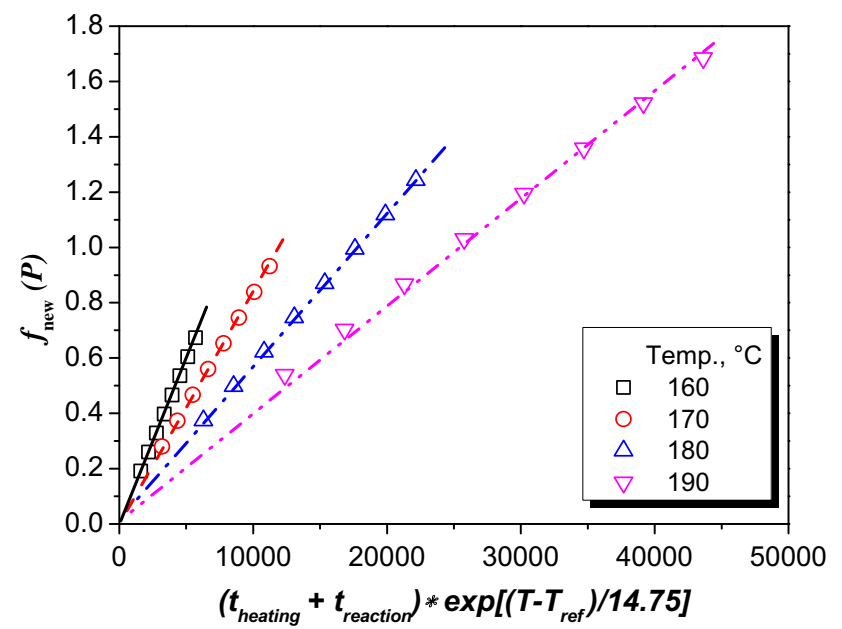

Fig. 4. Relationship between the traditional severity factor and the steam treatment factor.
$R_{0}=t \exp \left(\frac{T-T_{r}}{\omega}\right)$

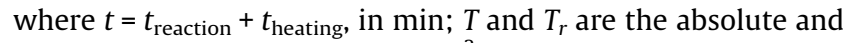
reference temperatures, in $\mathrm{K} ; \omega=R T_{r}^{2} E a=14.75$, and $T_{r}=373 \mathrm{~K}$.

Furthermore, the intercepts of $y$ were the same, and the differences were only in the slopes. According to the initial values of $T_{r}$ and $\omega$, the activation energy used in Eq. (21) could be estimated as $78.4 \mathrm{~kJ} / \mathrm{mol}$, which is higher than the corresponding value $(48.1 \mathrm{~kJ} / \mathrm{mol})$ obtained in Section 3.3. Therefore, the differences in the slopes were mainly contributed by the different activation energies used in Eqs. (19) and (21). Substitution of the activation energy obtained in Eq. (9) into Eq. (21) would give a new $\omega$ of 24.05. The data of $f_{\text {new }}(P)$ from each reaction temperature were found to lie on one curve (not shown) after substituting the new $\omega$ into Eq. (21). The $f_{\text {new }}(P)$ would be a simple form of $R_{0}$ if the same activation energy was adopted.

\subsection{Predicting the solid yield of green bamboo after steam treatment using $f_{\text {new }}(P)$}

The linear regression analysis conducted by Zhu et al. (2012a) suggested a robust correlation between the solid yield and the residual xylan during the sulfite-based pretreatment of aspen. They stated that the solid yield loss was mainly caused by the removal of hemicelluloses, whereas $30 \%$ of lignin loss and the minimal dissolution of cellulose could be attributed to the acidic pretreatment. Xylan removal is determined to be the major determinant of substrate enzymatic cellulose hydrolysis but not the only factor, since Klason lignin removal to the regression for substrate enzymatic digestibility (SED) in an Ftest is also significant. In this study, the linear correction coefficient $\left(R^{2}\right)$ between xylan removal and solids yield was determined to be 0.963 , indicating that there is also a strong correction between these two variations during steam treatment of green bamboo.

Based on the robust correlation of residual pentosan with $f_{\text {new }}(-$ $P$ ) as shown in Fig. 3(b), the data sets of measured solid yield were fitted using the Boltzmann function,

$Y=Y_{\min }+\frac{Y_{\max }-Y_{\min }}{1+\exp \left[\left(f_{\text {new }}(P)-f_{\text {new }}(P)_{0}\right) \cdot \Delta f_{\text {new }}(P)\right]}$

Origin 8.0 was employed for nonlinear curve fitting, and the parameters in Eq. (22) were obtained as $Y_{\max }=98.02, Y_{\min }=74.70$, $f_{\text {new }}(P)_{0}=0.61$, and $\Delta f_{\text {new }}(P)=0.29$. In contrast to the results reported by Zhu et al. (2012a), Eq. (22) obtained ideal fitting, as shown in Fig. 5 with $R^{2}=0.971$. This result could be because no catalysts were involved in the steam treatment, whereas two completely different catalysts were used in the study of Zhu et al. (2012a).

\subsection{Difference of steam and hot water treatment on pentosan removal}

The leading role of hydrothermal pretreatment on enhancing the efficiency of enzymatic hydrolysis is in making the hemicelluloses soluble, thereby making the cellulose more accessible to the cellulose microfibrils and avoiding the formation of inhibitors (Alvira et al., 2010; Kling et al., 1987; Ramos et al., 1992; Saddler et al., 1993; Tanaka and Matsuno, 1990). In addition to steam treatment, the use of hot water is another hydrothermal treatment. A uniform severity factor or steam treatment factor, $f(P)$, proposed in Section 3.4.1 could facilitate the comparison between the performances of steam treatment and hot water extraction for pentosan removal. In the combination of Eq. (20), the pentosan removal at time $\mathrm{t}\left(P R_{t}\right)$ during steam treatment was 


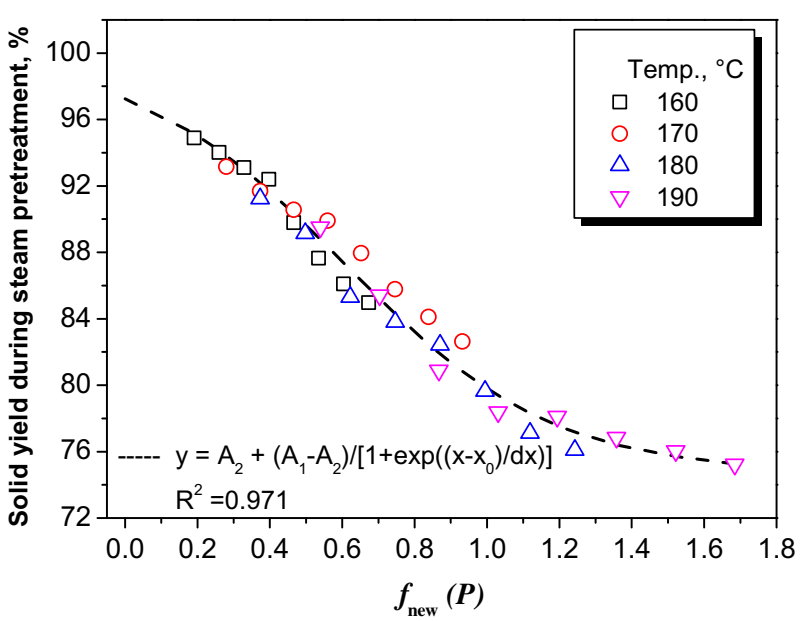

Fig. 5. Predicting the solid yield of green bamboo after steam treatment using $f_{\text {new }}(P)$.

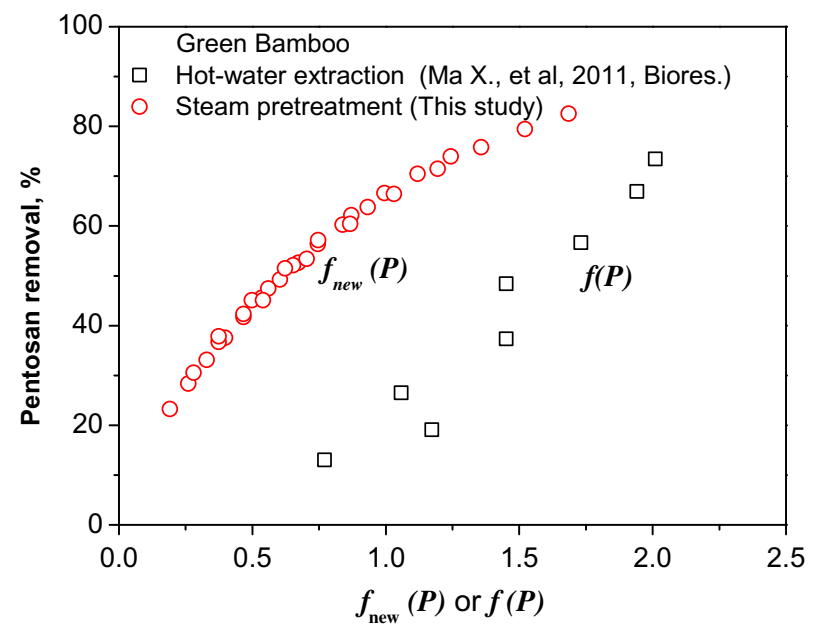

Fig. 6. Difference of steam and hot water treatments on pentosan removal.

$P R_{\mathrm{t}}=\left(1-\frac{R P}{P_{t_{0}}}\right) \times 100=\left(1-\frac{\beta}{e^{f_{\text {new }}(P)}}\right) \times 100$

The steam treatment factor was also used to estimate the reaction severity of hot water treatment and calculated by Eq. (18) due to its observable heating process. As shown in Fig. 6, the results clearly indicated that the steam treatment had better pentosan removing capability than that of hot water extraction under the same treatment severity. This finding could be attributed to the opening of the internal surface of bamboo due to better permeability of steam than that of liquid water, as well as the removal of more lignin that decreases the barrier of pentosan dissolution. Therefore, the efficiency for removing pentosan by steam treatment is higher than that of hot water extraction under the same reaction severity.

\section{Conclusions}

A simple first order kinetic model for overall pentosan solubility of green bamboo was developed. After deducted the fraction of soluble pentosan in heating-up period, parameters in kinetic model was effectively dissolved. Moreover, based on numerical algorithm method, pentosan solubility during heating process was also well modeled. To fit experimental data, classical kinetic model was modified, and a steam treatment factor $(f(P))$ was subsequently proposed. Finally, by introducing potential hydrolysis degree $\left(h_{\mathrm{d}}\right)$ and dissolved kinetic parameters into an exponential function, residual pentosan from the heating and reaction processes could be well fitted based on $f(P)$.

\section{Acknowledgements}

The authors gratefully acknowledge the financial support from JK Project of Fujian Provincial Department of Education (JK2012015), Open Fundation of the Chinese Academy of Sciences Key Laboratory of biofuels (No. CASKLB201308) and Open Fundation of the State Key Laboratory of Pulp and Paper Engineering (No. 201222). Doctoral Fund of Ministry of Education of China (No. 20123515120018). The authors also thank Dr. Xinsheng Chai (South China University of Technology, China) and Dr. Jing Liu (Waseda University, Japan; present in Fujian Agriculture and Forestry University (FAFU)) for their help in revising this manuscript.

\section{Appendix A. Supplementary data}

Supplementary data associated with this article can be found, in the online version, at http://dx.doi.org/10.1016/j.biortech.2012. 12.088 .

\section{References}

Abatzoglou, N., Chornet, E., Belkacemi, K., Overend, R.P., 1992. Phenomenological kinetics of complex systems: the development of a generalized severity parameter and its application to lignocellulosics fractionation. Chem. Eng. Sci. 47, 1109-1122.

Aguilar, R., Ramirez, J.A., Garrote, G., Vazquez, M., 2002. Kinetic study of the acid hydrolysis of sugar cane bagasse. J. Food Eng. 55, 309-318.

Alvira, P., Tomas, P.E., Ballesteros, M., Negro, M.J., 2010. Pretreatment technologies for an efficient bioethanol production process based on enzymatic hydrolysis: a review. Bioresour. Technol. 101, 4851-4861.

Arslan, Y., Saraçoğlu-Eken, N., 2012. Kinetic study of hemicellulosic sugar production from hazelnut shells. Chem. Eng. Sci. 185 (186), 23-28.

Brennan, M.A., Wyman, C.E., 2004. Initial evaluation of simple mass transfer models to describe hemicellulose hydrolysis in corn stover. Appl. Biochem. Biotechnol. 113-116, 965-976.

Chu, Y.M., 2005. Computer programming of bamboo fiber porosimetry. J. Bamboo Res. 24, 37-40.

Dekker, R.F.H., Karageorge, H., Wallis, A.F.A., 1987. Pretreatment of hardwood (Eucalyptus regnans) sawdust by autohydrolysis explosion and its saccharification by trichodermal cellulases. Biocatal. Biotransfor. 1, 47-61.

Garrote, G., Domínguez, H., Parajó, J.C., 2002. Interpretation of deacetylation and hemicellulose hydrolysis during hydrothermal treatments on the basis of the severity factor. Process Biochem. 37, 1067-1073.

Himmel, M.E., Ding, S.Y., Johnson, D.K., Adney, W.S., Nimlos, M.R., Brady, J.W., Foust, T.D., 2007. Biomass recalcitrance. engineering plants and enzymes for biofuels production. Science 315, 804-807.

Jensen, J., Morinelly, J., Aglan, A., Mix, A., Shonnard, D.R., 2008. Kinetic characterization of biomass dilute sulfuric acid hydrolysis: mixtures of hardwoods, softwood, and switchgrass. AIChE J. 54, 1637-1645.

Kling, S.H., Neto, C., Ferrara, M.A., Torres, J.C.R., Magalhaes, D.B., Ryu, D.D.Y., 1987. Enhancement of enzymatic hydrolysis of sugarcane bagasse by steam explosion pretreatment. Biotechnol. Bioeng. 29, 1035-1039.

Kobayashi, T., Sakai, Y., 1956. Hydrolysis rate of pentosan of hardwood in dilute sulfuric acid. Bull. Agric. Chem. Soc. Jpn. 20, 1-7.

Lavarack, B.P., Griffin, G.J., Rodman, D., 2000. Measured kinetics of the acid catalysed hydrolysis of sugar cane bagasse to produce xylose. Catal. Today 63, 257-265.

Levenspiel, O., 1999. Chemical Reaction Engineering, third ed., John Wiley \& Sons, pp. 386-391.

Lu, Y., Mosier, N.S., 2008. Kinetic modeling analysis of maleic acid-catalyzed hemicellulose hydrolysis in corn stover. Biotechnol. Bioeng. 101, 1170-1181.

Ma, X.J., Huang, L.L., Chen, Y.X., Cao, S.L., Chen, L.H., 2011. Preparation of bamboo dissolving pulp for textile production. Part 1 . Study on prehydrolysis of green bamboo for producing dissolving pulp. BioResources 6, 1428-1439.

Malm, C.J., Barkey, K.T., May, D.C., Lefferts, E.B., 1952. Treatment of cellulose prior to acetylation. Ind. Eng. Chem. 44, 2904-2909.

Maloney, M.T., Chapman, T.W., Baker, A.J., 1985. Dilute acid-hydrolysis of paper birch-kinetics studies of xylan and acetyl-group hydrolysis. Biotechnol. Bioeng. 27, 355-361.

Mason, W. H., 1929. Apparatus and process of explosion defibration of lignocellulosic material, U.S. Patent No.1655618. 
Mittal, A., Chatterjee, S.G., Scott, G.M., Amidon, T.A., 2009. Modeling xylan solubilization during autohydrolysis of sugar maple and aspen wood chips: reaction kinetics and mass transfer. Chem. Eng. Sci. 64, 3031-3041.

Ramos, L.P., Breuil, C., Kushner, D.N., Saddler, J.N., 1992. Steam pretreatment conditions for effective enzymatic hydrolysis and recovery yields of Eucalyptus viminalis wood chips. Holzforschung 46, 149-154.

Romero, I., Ruiz, E., Castro, E., Moya, M., 2010. Acid hydrolysis of olive tree biomass. Chem. Eng. Res. Des. 88, 633-640.

Saddler, J.N., Ramos, L.P., Breuil, C., 1993. Steam pretreatment of lignocellulosic residues. In: Saddler, J.N. (Ed.), Bioconversion of Forest and Agricultural Plant Wastes. CAB International, London, pp. 73-92.

Saeman, J.F., 1945. Kinetics of wood saccharification-hydrolysis of cellulose and decomposition of sugars in dilute acid at high temperature. Ind. Eng. Chem. 37, 43-52.

Sathitsuksanoh, N., Zhu, Z.G., Ho, T.J., Bai, M.D., Zhang, Y.H.P., 2010. Bamboo saccharification through cellulose solvent-based biomass pretreatment followed by enzymatic hydrolysis at ultra-low cellulase loadings. Bioresour. Technol. 101, 4926-4929.

Scurlock, J.M.O., Dayton, D.C., Hames, B., 2000. Bamboo: an overlooked biomass resource? Biomass Bioenergy 19, 229-244.

Shimokawa, T., Ishida, M., Yoshida, S., Nojiri, M., 2009. Effects of growth stage on enzymatic saccharification and simultaneous saccharification and fermentation of bamboo shoots for bioethanol production. Bioresour. Technol. 100, 6651-6654.
Springer, E.L., 1966. Hydrolysis os Aspenwood xylan with aqueous solutions of hydrochloric acid. TAPPI 49, 102-106.

Sundqvist, H., 2001. Modern kraft pulp mill: how to meet simultaneously economical, environmental and customers' requirements. Int. Pulp Week 12 46-51.

Tanaka, M., Matsuno, R., 1990. N-Butylamine and acid-steam explosion pretreatments of rice straw and hardwood: effects on substrate structure and enzymatic hydrolysis. Enzyme Microb. Techol. 12, 190-195.

Tsuda, M., Aoyama, M., Cho, N.S., 1998. Catalyzed steaming as pretreatment for the enzymatic hydrolysis of bamboo grass culms. Bioresour. Technol. 64, 241-243.

Vroom, K.E., 1957. A means of expressing cooking times and temperatures as a single variable. Pulp Paper Mag. Can. 58, 228-231.

Zhao, X.B., Zhou, Y.J., Liu, D.H., 2012. Kinetic model for glycan hydrolysis and formation of monosaccharides during dilute acid hydrolysis of sugarcane bagasse. Bioresour. Technol. 105, 160-168.

Zhu, W., Houtman, C.J., Zhu, J.Y., Gleisner, R., Chen, K.F., 2012a. Quantitative predictions of bioconversion of aspen by dilute acid and SPORL pretreatments using a unified combined hydrolysis factor (CHF). Process Biochem. 47, 785791.

Zhu, J.Y., Zhuang, X.S., 2012b. Conceptual net energy output for biofuel production from lignocellulosic biomass through biorefining. Prog. Energ. Combust. 38 583-598. 